Chirurg 2014 $\cdot 85: 1112$

DOI 10.1007/s00104-014-2910-z

Online publiziert: 27. November 2014

(c) Springer-Verlag Berlin Heidelberg 2014

O. Strobel · M.W. Büchler

Klinik für Allgemein-, Viszeral- und Transplantationschirurgie, Universität Heidelberg

\title{
Der postoperative Peak der Serum- transaminasen nach Leberresektion korreliert weder mit der Ischämiezeit noch mit der Morbidität
}

parameter waren Morbidität und Liegedauer. Multiple weitere Einflussparameter wie Indikation, Resektionsform und Durchführung einer vaskulären Okklusion wurden prospektiv erfasst. Einflussfaktoren auf PST und Morbidität wurden mittels multivariater Analysen untersucht.

\section{Hintergrund und Fragestellung}

Die Messung der Serumwerte der Transaminasen Aspartat-Aminotransferase $(\mathrm{AST} \triangleq \mathrm{GOT})$ und Alanin-Aminotransferase $(\mathrm{ALT} \triangleq \mathrm{GPT})$ ist gängige Praxis nach Leberresektionen, weil angenommen wird, dass die Peak-Werte der Serumtransaminasen (PST) mit dem ischämieinduzierten Leberzellschaden und mit dem postoperativen Outcome korrelieren. In zahlreichen klinischen Studien zur Leberchirurgie und insbesondere zur vaskulären Okklusion im Rahmen von Leberresektionen wurden PST daher als primärer Endpunkt benutzt. Allerdings wurde die tatsächliche Relevanz der PST nie formal bewiesen. Boleslawski et al. führten nun eine prospektive multizentrische Studie durch mit den Zielen, 1) den Einfluss einer vaskulären Okklusion auf die PST und 2) die Aussagekraft der PST auf das postoperative Outcome nach Leberresektionen zu untersuchen.

\section{Methoden}

Die beobachtende Studie wurde an 9 Zentren für Leberchirurgie in Frankreich von 01/2013 bis 09/2013 durchgeführt. Als primärer Zielparameter wurden die PST von AST und ALT gemessen. Sekundäre Ziel-

\section{Ergebnisse}

Es wurden 651 Patienten mit Leberresektion in die beobachtende Studie eingeschlossen. Eine Inflow-Okklusion wurde bei 58\% der Eingriffe durchgeführt (intermittierend in 32\%, kontinuierlich in 24\%). Die Morbidität betrug 50,8\%. Schwere Komplikationen traten bei $25,2 \%$ der Patienten auf. Die Mortalität lag bei 3,9\%. Die PST von AST und ALT traten am ersten postoperativen Tag auf und korrelierten nicht mit der Dauer der Inflow-Okklusion, sondern mit der Operationsdauer und der zusätzlichen Durchführung ablativer Maßnahmen. Im Gegensatz zu mehreren anderen Einflussfaktoren (BodyMass-Index, Diabetes, Zirrhose, Resektionsausmaß, präoperative Transaminasen und Bilirubin, Inflow-Okklusion, Bluttransfusion) waren die PST nicht unabhängig mit der Gesamtmorbidität oder dem Auftreten schwerer Komplikationen assoziiert. PST waren nicht geeignet, die Morbidität zuverlässig vorherzusagen.

\section{Fazit}

Die Studie zeigt eindeutig, dass die PST nicht mit der Ischämiezeit durch Inflow-
Okklusion korrelieren und als Surrogatmarker für das postoperative Ouctome nach Leberresektionen ungeeignet sind. Diese Erkenntnis hat eine hohe praktische Relevanz:

- Die gängige Praxis der Messung und Interpretation der Transaminasen nach Leberresektionen wird infrage gestellt. Hier müssen neue ergebnisrelevante Parameter entwickelt werden.

- Zusätzlich wird die klinische Relevanz zahlreicher Studien zur Bedeutung der intermittierenden und kontinuierlichen Inflow-Okklusion bei Leberresektionen infrage gestellt, die PST als primäre Zielparameter untersucht haben.

\section{Korrespondenzadresse}

\section{PD Dr. O. Strobel}

Klinik für Allgemein-, Viszeralund Transplantationschirurgie, Universität Heidelberg, Im Neuenheimer Feld 110, 69120 Heidelberg Oliver.Strobel@med.uni-heidelberg.de

Interessenkonflikt. O. Strobel und M.W. Büchler geben an, dass kein Interessenkonflikt besteht. 